\title{
Parity lifetime of bound states in a proximitized semiconductor nanowire
}

\author{
A. P. Higginbotham ${ }^{1,2 \uparrow}$, S. M. Albrecht ${ }^{1 \dagger}$, G. Kiršanskas ${ }^{1}$, W. Chang ${ }^{1,2}$, F. Kuemmeth ${ }^{1}$, P. Krogstrup ${ }^{1}$, \\ T. S. Jespersen ${ }^{1}$, J. Nygård ${ }^{1,3}{ }^{,}$K. Flensberg ${ }^{1}$ and C. M. Marcus ${ }^{1 \star}$
}

Quasiparticle excitations can compromise the performance of superconducting devices, causing high-frequency dissipation, decoherence in Josephson qubits ${ }^{1-6}$, and braiding errors in proposed Majorana-based topological quantum computers ${ }^{7-9}$. Quasiparticle dynamics have been studied in detail in metallic superconductors ${ }^{10-14}$ but remain relatively unexplored in semiconductor-superconductor structures, which are now being intensely pursued in the context of topological superconductivity. To this end, we use a system comprising a gate-confined semiconductor nanowire with an epitaxially grown superconductor layer, yielding an isolated, proximitized nanowire segment. We identify bound states in the semiconductor by means of bias spectroscopy, determine the characteristic temperatures and magnetic fields for quasiparticle excitations, and extract a parity lifetime (poisoning time) of the bound state in the semiconductor exceeding $10 \mathrm{~ms}$.

Semiconductor-superconductor hybrids have been investigated for many years ${ }^{15-19}$, but have received renewed interest as platforms for emergent topological superconductors with Majorana end modes. Such modes are expected to show non-Abelian statistics, allowing, in principle, topological encoding of quantum information $^{20-22}$ among other interesting effects ${ }^{23,24}$.

Transport experiments on semiconductor nanowires proximitized by a grounded superconductor have recently revealed characteristic signatures of Majorana modes ${ }^{25,26}$. Semiconductor quantum dots with superconducting leads have also been explored experimentally $y^{27-30}$, and have been proposed as a basis for Majorana chains $^{31-33}$. Here, we expand these geometries by creating an isolated semiconductor-superconductor hybrid quantum dot (HQD) connected to normal leads. The device forms the basis of an isolated, mesoscopic Majorana system with protected total parity ${ }^{34,35}$.

The measured device consists of a hexagonal InAs nanowire with epitaxial superconducting $\mathrm{Al}$ on two facets ${ }^{36,37}$, and Au ohmic contacts (Fig. 1a,b), forming a normal metal-superconductornormal metal (NSN) device. Four devices showing similar behaviour have been measured. Differential conductance, $g$, was measured in a dilution refrigerator $(T \sim 50 \mathrm{mK})$ using standard lock-in techniques. Local side gates and a global back gate were adjusted to form an Al-InAs HQD in the Coulomb blockade regime. The lower right gate, $V_{\mathrm{R}}$, was used to tune the occupation of the dot, with a linear compensation from the lower left gate, $V_{\mathrm{L}}$, to keep tunnelling to the leads symmetric. We parameterize this with a single effective gate voltage, $V_{\mathrm{G}}$ (see Supplementary Information).

Differential conductance as a function of $V_{\mathrm{G}}$ and source-drain bias, $V_{\mathrm{SD}}$, reveals a series of Coulomb diamonds, corresponding a

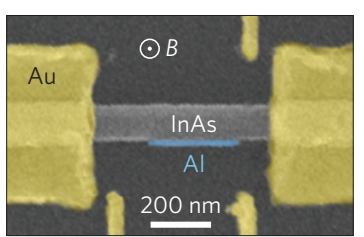

b

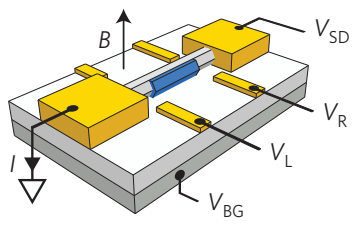

C

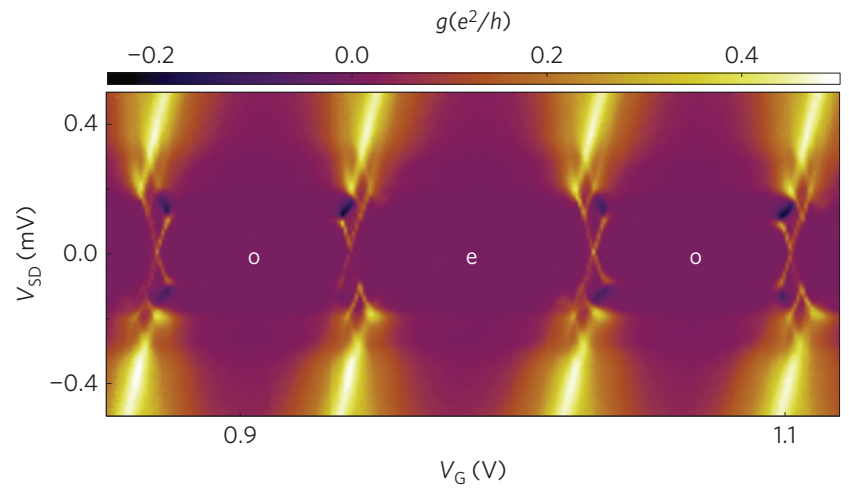

Figure 1 | Nanowire-based hybrid quantum dot. a, Scanning electron micrograph of the reported device, consisting of an InAs nanowire (grey) with a segment of epitaxial Al on two facets (blue) and Ti/Au contacts and side gates (yellow) on a doped silicon substrate with $100 \mathrm{~nm}$ oxide. b, Device schematic and measurement set-up, showing the orientation of the magnetic field, $B$. c, Differential conductance, $g$, as a function of effective gate voltage, $V_{\mathrm{G}}$, and source-drain voltage, $V_{\mathrm{SD}}$, at $B=0$. Even (e) and odd (o) occupied Coulomb valleys are labelled.

to incremental single-charge states of the HQD (Fig. 1c). Whereas conductance features at high bias are essentially identical in each diamond, at low bias, $V_{\mathrm{SD}}<0.2 \mathrm{mV}$, a repeating even-odd pattern of left- and right-facing conductance features is observed. This results in an even-odd alternation of Coulomb blockade peak spacings at zero bias, similar to metallic superconductors ${ }^{38,39}$. However, the parity-dependent reversing pattern of subgap features at non-zero bias has not been reported before, to our knowledge. The repeating even-odd pattern indicates that a parity-sensitive bound state is being repeatedly filled and emptied as electrons are added to the HQD.

The measured charging energy, $E_{\mathrm{C}}=1.1 \mathrm{meV}$, and superconducting gap, $\Delta=180 \mu \mathrm{eV}$, satisfy the condition $\left(\Delta<E_{\mathrm{C}}\right)$ for single electron charging ${ }^{40,41}$. Differential conductance at low

${ }^{1}$ Center for Quantum Devices and Station Q-Copenhagen, Niels Bohr Institute, University of Copenhagen, 2100 Copenhagen, Denmark. ${ }^{2}$ Department of Physics, Harvard University, Cambridge, Massachusetts 02138, USA. ${ }^{3}$ Nano-Science Center, Niels Bohr Institute, University of Copenhagen,

2100 Copenhagen, Denmark. †These authors contributed equally to this work. ${ }^{\star} e-m a i l:$ marcus@nbi.dk 
bias occurs in a series of narrow features symmetric about zero bias, suggesting transport through a bound state, with negative differential conductance (NDC) observed at the border of odd diamonds. NDC arises from slow quasiparticle escape, similar to current blocking seen in metallic superconducting islands in the opposite regime, $\Delta>E_{\mathrm{C}}$ (refs 42,43 ).

We present a simple model of transport through a single bound state in the InAs plus a Bardeen-Cooper-Schrieffer (BCS) continuum in the Al. The model makes several simplifying assumptions: a fixed-energy bound state, motivated by the repetitive pattern observed in the Coulomb diamonds, and symmetric coupling of both the bound state and continuum to the leads, motivated by the observed symmetry in $V_{\mathrm{SD}}$ of the Coulomb diamonds. Transition rates were calculated from Fermi's golden rule and a steady-state Pauli master equation was solved for state occupancies. Conductance was then calculated from occupancies and transition rates (see Supplementary Information).

Measured and model conductances are compared in Fig. 2a,b. The coupling of the bound state to each lead, noting the nearsymmetry of the diamonds, was estimated to be $\Gamma_{0}=0.5 \mathrm{GHz}$, based on zero-bias conductance (Fig. 2d). The energy of the state, $E_{0}=58 \mu \mathrm{eV}$ at zero magnetic field, was measured using finite bias spectroscopy (Fig. 2e). The normal-state conductance from each lead to the continuum, $g_{\mathrm{Al}}=0.15 e^{2} / h$, was estimated by comparing Coulomb blockaded transport features in the high-bias regime $\left(V_{\mathrm{SD}}=0.4 \mathrm{mV}\right.$ ). The superconducting gap, $\Delta=180 \mu \mathrm{eV}$, was found from the onset of NDC at $e V_{\mathrm{SD}}=\Delta-E_{0}$ (Fig. 2f). Although the rate model shows good agreement with experimental data, some features are not captured, including broadening at high bias, with greater broadening correlated with weaker NDC, and peak-to-peak fluctuations in the slope of the NDC feature. These features may be related to heating or co-tunnelling, not accounted for by the model.

The observation of negative differential conductance places a bound on the relaxation rate of a single quasiparticle in the HQD from the continuum (in the $\mathrm{Al}$ ) to the bound state (in the InAs nanowire). NDC arises when an electron tunnels into the weakly coupled BCS continuum, blockading transport until it exits via the lead. The blocking condition is shown for a holelike excitation in Fig. 2f. Unblocking occurs when the quasiparticle relaxes into the bound state, followed by a fast escape to the leads. NDC thus indicates a long quasiparticle relaxation time, $\tau_{\mathrm{qp}}$, from the continuum to the bound state. Using independently determined parameters, the observed NDC is compatible with the model only when $\tau_{\mathrm{qp}}>0.1 \mu$ s (see Supplementary Information), which is used below to constrain the poisoning time for the bound state.

Turning our attention to the even-odd structure at zero bias, we observed consistent large-small peak spacings (Fig. 3a,b), associating larger spacings with even occupation, as expected theoretically ${ }^{40,41}$. Parity reversals were observed on the timescale of hours, similar to observations in metallic devices ${ }^{14}$. Peak spacing alternation disappears at higher magnetic fields, $B$, consistent with the superconducting-to-normal transition, and also disappears at elevated temperature, $T>0.4 \mathrm{~K}$, significantly below the superconducting critical temperature, $T_{\mathrm{c}} \sim 1 \mathrm{~K}$. The temperature dependence is similar to metallic structures ${ }^{38,39}$, and can be understood as the result of thermal activation of quasiparticles within the HQD with fixed total charge.

As seen in Fig. 3c, individual Coulomb peaks are asymmetric in shape, with their centroids (first moments) on the even sides of the peak maxima. The asymmetric shape is most pronounced at low temperature, $T<0.15 \mathrm{~K}$, and decreases with increasing magnetic field. The degree of asymmetry is not predicted by the rate model, even taking into account the known small asymmetry due to spin degeneracy ${ }^{44}$. In the analysis below, we consider peak positions defined both by peak maxima and centroids. a

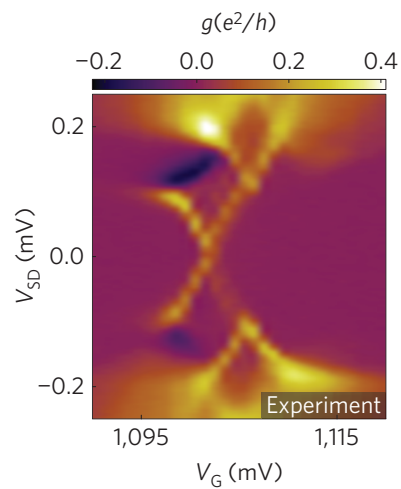

b
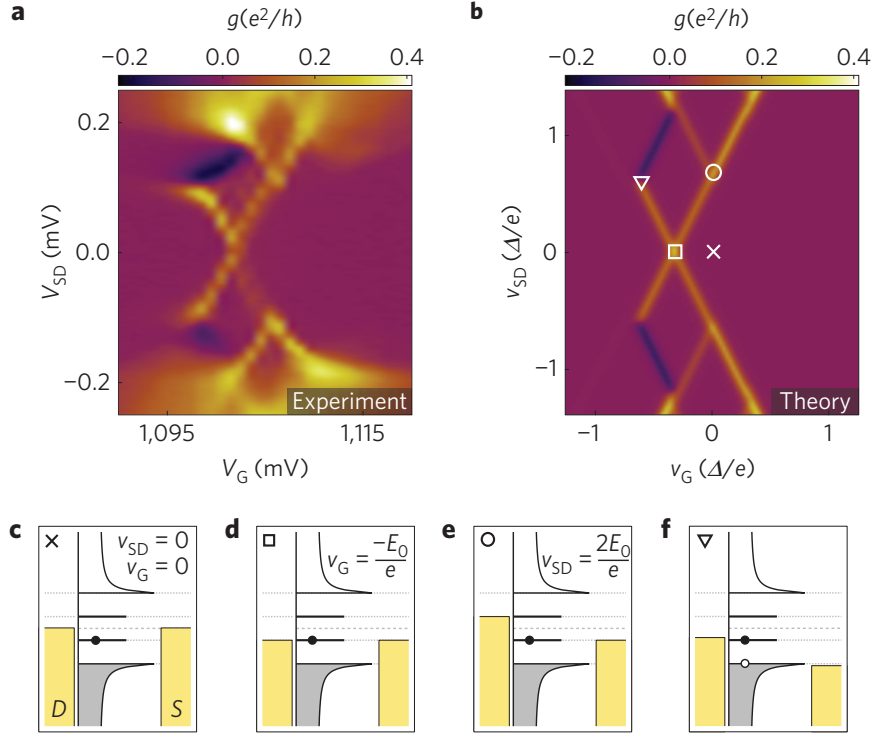

Figure 2 | Subgap bias spectroscopy, experiment and model.

a, Experimental differential conductance, $g$, as a function of gate voltage $V_{G}$ and source-drain bias $V_{S D}$, shows a characteristic pattern including NDC.

b, Transport model of a. $v_{\mathrm{G}}=\alpha V_{\mathrm{G}}$ up to an offset, where $\alpha$ is the gate lever arm. Axis units are $\Delta / e=180 \mu \mathrm{V}$, where $\Delta$ is the superconducting gap. See text for model parameters. c, Source and drain (gold) chemical potentials align with the middle of the gap in the HQD density of states. No transport occurs owing to the presence of superconductivity. d, Discrete state in resonance with the leads at zero bias. Transport occurs through single quasiparticle states. e, Discrete state in resonance with the leads at high bias. Transport occurs through single and double (particle-hole) quasiparticle states. f, Discrete state and BCS continuum in the bias window. Transport is blocked when a quasiparticle is in the continuum, resulting in NDC.

A model of even-odd Coulomb peak spacing that includes thermal quasiparticle excitations follows earlier treatments ${ }^{38,39,41}$, including a discrete subgap state as well as the BCS continuum ${ }^{39}$ (Fig. 3d). The even-odd peak spacing difference, $S_{\mathrm{e}}-S_{\mathrm{o}}$, depends on the difference of free energies,

$$
S_{\mathrm{e}}-S_{\mathrm{o}}=\frac{4}{\alpha e}\left(F_{\mathrm{o}}-F_{\mathrm{e}}\right)
$$

where $\alpha$ is the (dimensionless) gate lever arm. The free energy difference, written in terms of the ratio of the partition functions $Z$,

$$
F_{\mathrm{o}}-F_{\mathrm{e}}=-k_{\mathrm{B}} T \ln \left(\frac{Z_{\mathrm{o}}}{Z_{\mathrm{e}}}\right)
$$

depends on $D(E)$, the density of states of the HQD,

$$
\frac{Z_{\mathrm{o}}}{Z_{\mathrm{e}}}=\int_{0}^{\infty} \mathrm{d} E D(E) \ln \operatorname{coth}\left[E /\left(2 k_{\mathrm{B}} T\right)\right]
$$

where $D(E)$ consists of one subgap state and the continuum. For $\Delta \gg k_{\mathrm{B}} T$, this can be written

$$
F_{\mathrm{o}}-F_{\mathrm{e}} \approx-k_{\mathrm{B}} T \ln \left(N_{\mathrm{eff}} \mathrm{e}^{-\Delta / k_{\mathrm{B}} T}+2 \mathrm{e}^{-E_{0} / k_{\mathrm{B}} T}\right)
$$

where $N_{\text {eff }}=\rho_{\mathrm{Al}} V_{\mathrm{Al}} \sqrt{2 \pi k_{\mathrm{B}} T \Delta}$ is the effective number of continuum states for $\mathrm{Al}$ with volume $V_{\mathrm{Al}}$ and electron density of states $\rho_{\mathrm{Al}}$ (refs 38,39).

Within the thermodynamic model, one can identify a characteristic temperature, $T^{*} \sim \Delta /\left[k_{\mathrm{B}} \ln \left(N_{\text {eff }}\right)\right]$, less than the gap and independent of $E_{0}$, above which even-odd peak spacing 

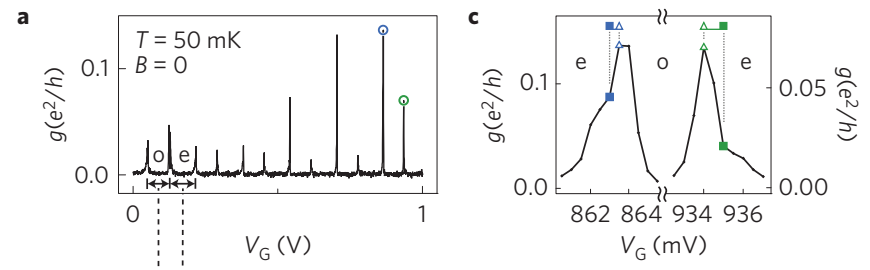

b
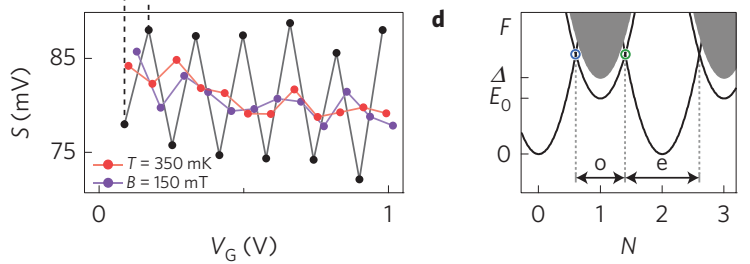

Figure 3 | Even-odd Coulomb peak spacings. a, Measured zero-bias conductance, $g$, versus gate voltage, $V_{\mathrm{G}}$, at temperature $T \sim 50 \mathrm{mK}$ and magnetic field $B=0$. b. Peak spacing, $S$, versus gate voltage. Black points show spacings from a calculated using the peak centroid (first moment), red points $T=350 \mathrm{mK}$ and $B=0$, purple points $B=150 \mathrm{mT}$ and $T \sim 50 \mathrm{mK}$. c, Rightmost peaks in a. Peak maxima (open triangle) and centroids (filled square) are marked. d, Free energy, $F$, at $T=0$ versus gate-induced charge, $N$, for different HQD occupations, where $N=C V_{G} / e$ up to an offset and $C$ is the gate capacitance. Parabola intersection points are indicated by circles, corresponding to Coulomb peaks. BCS continuum (shaded), shown for odd occupancy. Odd Coulomb diamonds carry an energy offset $E_{0}$ for quasiparticle occupation of the subgap state, resulting in a difference in spacing for even and odd diamonds.

alternation is expected to disappear. A second (lower) characteristic temperature, $T^{* *} \sim\left(\Delta-E_{0}\right) /\left[k_{\mathrm{B}} \ln \left(N_{\text {eff }} / 2\right)\right]$, is where the even-odd alternation is affected by the bound state, leading to saturation at low temperature ${ }^{38,39}$. For a zero-energy $\left(E_{0}=0\right)$ bound state-the case for Majorana modes-these characteristic temperatures coincide and even-odd structure vanishes, as pointed out in ref. 34. For $E_{0}=\Delta$ the saturation temperature vanishes, $T^{* *}=0$, and the metallic result is recovered ${ }^{38,39}$.

Experimentally, the average even-odd peak spacing difference, $\left\langle S_{\mathrm{e}}-S_{\mathrm{o}}\right\rangle$, was determined by averaging over a set of 24 consecutive Coulomb peak spacings, including those shown in Fig. 3. Figure 4 shows the even-odd peak spacing difference appearing abruptly at $T_{\text {onset }} \sim 0.4 \mathrm{~K}$, and saturating at $T_{\text {sat }} \sim 0.2 \mathrm{~K}$, with a saturation amplitude near the value expected from the measured boundstate energy, $4 V_{0}=4 E_{0} /(\alpha e)$. Figure 4 shows good agreement between experiment and the model, equation (1), using a density of states determined independently from data in Fig. 2, with $V=7.4 \times 10^{4} \mathrm{~nm}^{3}$ as a fit parameter, consistent with the micrograph (Fig. 1a), and $\rho_{\mathrm{Al}}=23 \mathrm{eV}^{-1} \mathrm{~nm}^{-3}$ (ref. 14).

The asymmetric peak shape amounts to larger peak tails on the even valley side, causing the centroids to be more regularly spaced than the maxima. This is evident in Fig. 4, where the centroid method shows a decreasing peak spacing difference at low temperature, whereas with the maximum method the spacing remains flat. The thermal model of $S_{\mathrm{e}}-S_{\mathrm{o}}$ can also show a decrease at low temperature if broadening of the bound state is included. We do not understand at present if this effect explains the difference between centroids and maxima, however, it is worth noting that the fit gives a broadening $\gamma=50 \mathrm{neV}$, reasonably close to the value estimated from the lead couplings, $\left(h \Gamma_{0}\right)^{2} / \Delta=20 \mathrm{neV}$.

An applied magnetic field (direction shown in Fig. 1b) reduces the characteristic temperatures $T_{\text {onset }}, T_{\text {sat }}$, and saturation amplitudes. Field dependence is modelled by including Zeeman splitting of the bound state and orbital reduction of the gap. The fit $g$-factor, $g=6$, lies within the typical range for InAs

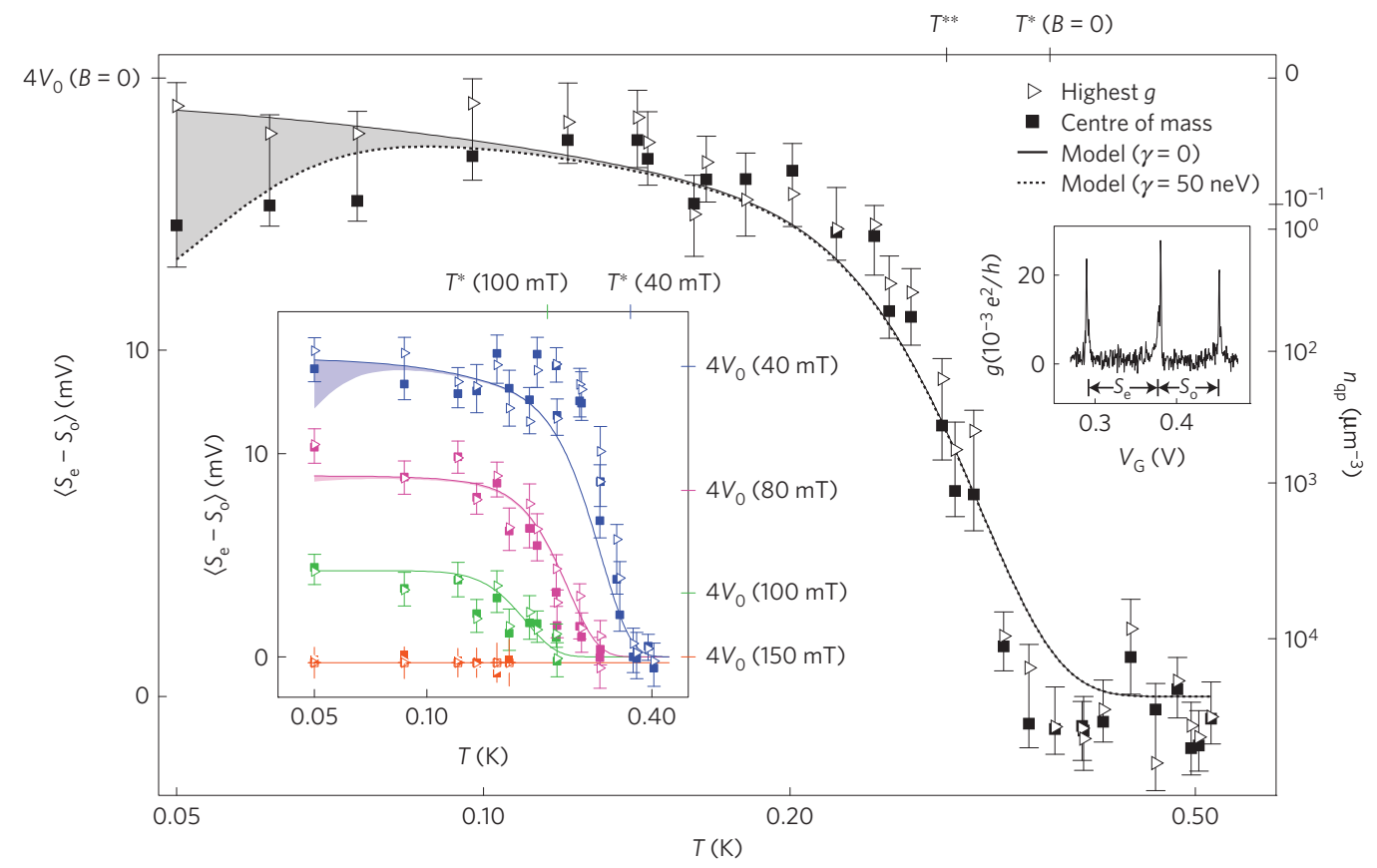

Figure 4 | Temperature and magnetic field dependence of the even-odd peak spacings. Average even-odd spacing difference, $\left\langle S_{e}-S_{0}\right\rangle$, versus temperature, $T$. Spacings between peak maxima (triangle) and centroids (square) are shown, with error bars from the standard deviation of peak maxima. Spacings expected from lower Zeeman-split bound state, $4 V_{0}(B)=4 E_{0}(B) /(\alpha e)$ are indicated. Quasiparticle activation temperature, $T^{*}$, and crossover temperature, $T^{* *}$, indicated on top axis. Right axis shows the calculated aluminium quasiparticle density, $n_{\mathrm{qp}}$ (see text). Solid curve is equation (1) with a HQD density of states measured from Fig. $2\left(\Delta=180 \mu \mathrm{eV}, E_{0}=58 \mu \mathrm{eV}, \alpha=0.013\right)$, and the fitted aluminium volume, $V_{\mathrm{Al}}=7.4 \times 10^{4} \mathrm{~nm}^{3}$. Dotted curve includes a discrete state broadening, $\gamma=50 \mathrm{neV}$, fitted to the centroid data. Left inset: same as main figure, but at $B=40,80,100$ and $150 \mathrm{mT}$, from top to bottom and $4 V_{0}$ appearing on the right-hand axis. Curves are fitted to two shared parameters: $g$-factor, $g=6$, and superconducting critical field, $B_{c}=120 \mathrm{mT}$, with the other parameters fixed from the main figure. Right inset: representative Coulomb peaks showing even $\left(S_{e}\right)$ and odd $\left(S_{0}\right)$ spacings. 
nanowires ${ }^{45,46}$, supporting our interpretation that the bound state resides in the InAs.

Agreement with the thermodynamic model suggests that ensemble averages of even-odd spacing, $S_{\mathrm{e}}-S_{\mathrm{o}}$, provide a measure of the equilibrium quasiparticle density, $n_{\mathrm{qp}}$. Figure 4 (right axis) gives the value $n_{\mathrm{qp}}(T)=V_{\mathrm{Al}}^{-1} N_{\mathrm{eff}}^{2} \mathrm{e}^{-2 \Delta / k_{\mathrm{B}} T}$, an expression valid for large charging energy ${ }^{47}$ (see Supplementary Methods and Supplementary Section 5 ). Below $T_{\text {sat }} \sim 0.2 \mathrm{~K}$, even-odd spacing saturates at the bound-state value $4 V_{0}$, making it difficult to infer a quasiparticle density in this low-temperature range. Instead, we conservatively take $n_{\mathrm{qp}}\left(T_{\mathrm{sat}}\right) \sim 0.1 \mu \mathrm{m}^{-3}$ as an upper bound for the quasiparticle density at low temperature. This value is within the range from the recent literature, $0.03-30 \mu \mathrm{m}^{-3}$ (refs 3-6,13). Because the volume of $\mathrm{Al}$ is small, the upper bound on the number of quasiparticles, $n_{\mathrm{qp}} V_{\mathrm{Al}}<10^{-5}$, is, correspondingly, small.

Finally, we determine a lower bound on the poisoning time, $\tau_{\mathrm{p}}$, of the bound state. The physical mechanism for this poisoning is relaxation of a quasiparticle into the InAs from the $\mathrm{Al}$, which preserves the overall parity of the HQD but changes the parity of the bound state. This process is expected to set the fundamental limit on parity lifetime ${ }^{8}$. The poisoning rate, $1 / \tau_{\mathrm{p}}$, is given by the product of the relaxation rate of a single quasiparticle from the $\mathrm{Al}$, $1 / \tau_{\mathrm{qp}}$, and the number of quasiparticles in the $\mathrm{Al}$ (ref. 8), which, from above, is bounded by $n_{\mathrm{qp}} V_{\mathrm{Al}}<10^{-5}$. Quantitative analysis of the strength of negative differential conductance at finite bias-which vanishes for fast quasiparticle relaxation-provides a lower bound on the quasiparticle relaxation time, $\tau_{\mathrm{qp}}>0.1 \mu$ s (Supplementary Section 2). Together, these values give a conservative lower bound on the poisoning time of the bound state, $\tau_{\mathrm{p}}=\tau_{\mathrm{qp}} /\left(n_{\mathrm{qp}} V_{\mathrm{Al}}\right)>10 \mathrm{~ms}$.

Quasiparticle density depends sensitively on device geometry, filtering and shielding, resulting in a wide range of experimental values $\left(0.03-30 \mu \mathrm{m}^{-3}\right.$; refs $\left.3-6\right)$, and thus poisoning times. We note that recent work in transmon qubits ${ }^{13}$ found $n_{\mathrm{qp}}=0.04 \mu \mathrm{m}^{-3}$, corresponding to state-poisoning times well above $10 \mathrm{~ms}$. We also note that the Coulomb blockade geometry effectively enforces quasiparticles from the Al shell to be created only in pairs, which is different from non-charging device geometries.

On the basis of previous work, $\tau_{\mathrm{qp}}$, hence $\tau_{\mathrm{p}}$, is expected to depend weakly on the bound-state energy for low-energy bound states $^{11,48,49}$, including zero-energy Majorana modes with $E_{0}=0$. The long poisoning time found here, $\tau_{\mathrm{p}}>10 \mathrm{~ms}$, is auspicious for application of this system to topological quantum computing, suggesting that a large number of braiding operations of Majorana modes could be performed before the parity of the bound state is poisoned by the proximitizing Al. Future work will examine Majorana modes in this geometry.

\section{Methods}

Methods and any associated references are available in the online version of the paper.

Received 12 January 2015; accepted 4 August 2015; published online 14 September 2015

\section{References}

1. Lang, K. M., Nam, S., Aumentado, J., Urbina, C. \& Martinis, J. M. Banishing quasiparticles from Josephson-junction qubits: Why and how to do it. IEEE Trans. Appl. Supercond. 13, 989-993 (2003).

2. Aumentado, J., Keller, M. W., Martinis, J. M. \& Devoret, M. H. Nonequilibrium quasiparticles and $2 e$ periodicity in single-Cooper-pair transistors. Phys. Rev. Lett. 92, 066802 (2004)

3. Martinis, J. M., Ansmann, M. \& Aumentado, J. Energy decay in superconducting Josephson-junction qubits from nonequilibrium quasiparticle excitations. Phys. Rev. Lett. 103, 097002 (2009).

4. De Visser, P. J. et al. Number fluctuations of sparse quasiparticles in a superconductor. Phys. Rev. Lett. 106, 167004 (2011).
5. Saira, O. P., Kemppinen, A., Maisi, V. F. \& Pekola, J. P. Vanishing quasiparticle density in a hybrid $\mathrm{Al} / \mathrm{Cu} / \mathrm{Al}$ single-electron transistor. Phys. Rev. B 85, 012504 (2012)

6. Pop, I. M. et al. Coherent suppression of electromagnetic dissipation due to superconducting quasiparticles. Nature 508, 369-372 (2014).

7. Leijnse, M. \& Flensberg, K. Scheme to measure Majorana fermion lifetimes using a quantum dot. Phys. Rev. B 84, 140501 (2011).

8. Rainis, D. \& Loss, D. Majorana qubit decoherence by quasiparticle poisoning. Phys. Rev. B 85, 174533 (2012).

9. Cheng, M., Lutchyn, R. M. \& Das Sarma, S. Topological protection of Majorana qubits. Phys. Rev. B 85, 165124 (2012).

10. Ferguson, A., Court, N., Hudson, F. \& Clark, R. Microsecond resolution of quasiparticle tunneling in the single-Cooper-pair transistor. Phys. Rev. Lett. 97, 106603 (2006)

11. Zgirski, M. et al. Evidence for long-lived quasiparticles trapped in superconducting point contacts. Phys. Rev. Lett. 106, 257003 (2011).

12. Sun, L. et al. Measurements of quasiparticle tunneling dynamics in a band-gap-engineered transmon qubit. Phys. Rev. Lett. 108, 230509 (2012).

13. Ristè, D. et al. Millisecond charge-parity fluctuations and induced decoherence in a superconducting transmon qubit. Nature Commun. 4, 1913 (2013).

14. Maisi, V. F. et al. Excitation of single quasiparticles in a small superconducting $\mathrm{Al}$ island connected to normal-metal leads by tunnel junctions. Phys. Rev. Lett. 111, 147001 (2013).

15. Doh, Y. J. et al. Tunable supercurrent through semiconductor nanowires. Science 309, 272-275 (2005).

16. Hofstetter, L., Csonka, S., Nygård, J. \& Schönenberger, C. Cooper pair splitter realized in a two-quantum-dot Y-junction. Nature 461, 960-963 (2009).

17. Pillet, J.-D. et al. Andreev bound states in supercurrent-carrying carbon nanotubes revealed. Nature Phys. 6, 965-969 (2010).

18. De Franceschi, S., Kouwenhoven, L., Schönenberger, C. \& Wernsdorfer, W. Hybrid superconductor-quantum dot devices. Nature Nanotech. 5, 703-711 (2010)

19. Giazotto, F. et al. A Josephson quantum electron pump. Nature Phys. 7, 857-861 (2011)

20. Sau, J. D., Lutchyn, R. M., Tewari, S. \& Das Sarma, S. Generic new platform for topological quantum computation using semiconductor heterostructures. Phys. Rev. Lett. 104, 040502 (2010).

21. Lutchyn, R. M., Sau, J. D. \& Das Sarma, S. Majorana fermions and a topological phase transition in semiconductor-superconductor heterostructures. Phys. Rev. Lett. 105, 077001 (2010).

22. Oreg, Y., Refael, G. \& von Oppen, F. Helical liquids and Majorana bound states in quantum wires. Phys. Rev. Lett. 105, 177002 (2010).

23. Padurariu, C. \& Nazarov, Y. V. Theoretical proposal for superconducting spin qubits. Phys. Rev. B 81, 144519 (2010).

24. Leijnse, M. \& Flensberg, K. Coupling spin qubits via superconductors. Phys. Rev. Lett. 111, 060501 (2013).

25. Mourik, V. et al. Signatures of Majorana fermions in hybrid superconductor-semiconductor nanowire devices. Science 336, 1003-1007 (2012).

26. Das, A. et al. Zero-bias peaks and splitting in an Al-InAs nanowire topological superconductor as a signature of Majorana fermions. Nature Phys. 8, 887-895 (2012)

27. Deacon, R. S. et al. Tunneling spectroscopy of Andreev energy levels in a quantum dot coupled to a superconductor. Phys. Rev. Lett. 104, 076805 (2010).

28. Lee, E. J. H. et al. Zero-bias anomaly in a nanowire quantum dot coupled to superconductors. Phys. Rev. Lett. 109, 186802 (2012).

29. Lee, E. J. H. et al. Spin-resolved Andreev levels and parity crossings in hybrid superconductor-semiconductor nanostructures. Nature Nanotech. 9, 79-84 (2014)

30. Chang, W., Manucharyan, V. E., Jespersen, T. S., Nygård, J. \& Marcus, C. M. Tunneling spectroscopy of quasiparticle bound states in a spinful Josephson junction. Phys. Rev. Lett. 110, 217005 (2013).

31. Sau, J. D. \& Das Sarma, S. Realizing a robust practical Majorana chain in a quantum-dot-superconductor linear array. Nature Commun. 3, 964 (2012).

32. Leijnse, M. \& Flensberg, K. Parity qubits and poor man's Majorana bound states in double quantum dots. Phys. Rev. B 86, 134528 (2012).

33. Fulga, I. C., Haim, A., Akhmerov, A. R. \& Oreg, Y. Adaptive tuning of Majorana fermions in a quantum dot chain. New J. Phys. 15, 045020 (2013).

34. Fu, L. Electron teleportation via Majorana bound states in a mesoscopic superconductor. Phys. Rev. Lett. 104, 056402 (2010).

35. Hützen, R., Zazunov, A., Braunecker, B., Yeyati, A. L. \& Egger, R. Majorana single-charge transistor. Phys. Rev. Lett. 109, 166403 (2012).

36. Krogstrup, P. et al. Epitaxy of semiconductor-superconductor nanowires. Nature Mater. 14, 400-406 (2015).

37. Chang, W. et al. Hard gap in epitaxial semiconductor-superconductor nanowires. Nature Nanotech. 10, 232-236 (2015). 
38. Tuominen, M. T., Hergenrother, J. M., Tighe, T. S. \& Tinkham, M. Experimental evidence for parity-based $2 e$ periodicity in a superconducting single-electron tunneling transistor. Phys. Rev. Lett. 69, 1997-2000 (1992).

39. Lafarge, P., Joyez, P., Esteve, D., Urbina, C. \& Devoret, M. H. Measurement of the even-odd free-energy difference of an isolated superconductor. Phys. Rev. Lett. 70, 994-997 (1993).

40. Averin, D. V. \& Nazarov, Y. V. Single-electron charging of a superconducting island. Phys. Rev. Lett. 69, 1993-1996 (1992).

41. Tinkham, M. Introduction to Superconductivity 2nd edn (Dover, 2004).

42. Hekking, F. W. J., Glazman, L. I., Matveev, K. A. \& Shekhter, R. I. Coulomb blockade of two-electron tunneling. Phys. Rev. Lett. 70, 4138-4141 (1993).

43. Hergenrother, J. M., Tuominen, M. T. \& Tinkham, M. Charge transport by Andreev reflection through a mesoscopic superconducting island. Phys. Rev. Lett. 72, 1742-1745 (1994).

44. Aleiner, I. L., Brouwer, P. W. \& Glazman, L. I. Quantum effects in Coulomb blockade. Phys. Rep. 358, 309-440 (2002).

45. Björk, M. T. et al. Tunable effective $g$ factor in InAs nanowire quantum dots. Phys. Rev. B 72, 201307 (2005).

46. Csonka, S. et al. Giant fluctuations and gate control of the $g$-factor in InAs nanowire quantum dots. Nano Lett. 8, 3932-3935 (2008).

47. Matveev, K. A., Glazman, L. I. \& Shekhter, R. I. Effects of charge parity in tunneling through a superconducting grain. Mod. Phys. Lett. 08, 1007-1026 (1994).

48. Olivares, D. G. et al. Dynamics of quasiparticle trapping in Andreev levels. Phys. Rev. B 89, 104504 (2014).
49. Zazunov, A., Brunetti, A., Yeyati, A. L. \& Egger, R. Quasiparticle trapping, Andreev level population dynamics, and charge imbalance in superconducting weak links. Phys. Rev. B 90, 104508 (2014).

\section{Acknowledgements}

We thank L. Glazman, B. Halperin, R. Lutchyn and J. Pekola for valuable discussions, and G. Ungaretti, S. Upadhyay and C. Sørensen for contributions to growth and fabrication. Research support by Microsoft Project Q, the Danish National Research Foundation, the Lundbeck Foundation, the Carlsberg Foundation, and the European Commission. A.P.H. acknowledges support from the US Department of Energy, C.M.M. acknowledges support from the Villum Foundation.

\section{Author contributions}

P.K., T.S.J. and J.N. developed the nanowire materials. S.M.A. fabricated the devices, A.P.H., S.M.A. and W.C. carried out the measurements with input from F.K., T.S.J. and C.M.M. The theoretical model was developed by G.K., K.F. and A.P.H. All authors contributed to analysing and interpreting the data and to writing the manuscript.

\section{Additional information}

Supplementary information is available in the online version of the paper. Reprints and permissions information is available online at www.nature.com/reprints.

Correspondence and requests for materials should be addressed to C.M.M.

\section{Competing financial interests}

The authors declare no competing financial interests. 


\section{Methods}

Sample preparation. InAs nanowires were grown without stacking faults in the [001] direction with a wurtzite crystal structure with Al epitaxially matched to [111] on two of the six $\{1 \overline{1} 00\}$ side facets ${ }^{36,37}$. They were then deposited randomly onto a doped silicon substrate with $100 \mathrm{~nm}$ of thermal oxide. Electron-beam lithographically patterned wet etch of the epitaxial Al shell (Transene Al Etchant D, $55 \mathrm{C}, 10 \mathrm{~s}$ ) resulted in a submicron $\mathrm{Al}$ segment $(310 \mathrm{~nm}$, Fig. 1a). Ti/Au $(5 / 100 \mathrm{~nm})$ ohmic contacts were deposited on the ends following in situ Ar milling ( 1 mtorr, $300 \mathrm{~V}, 75 \mathrm{~s}$ ), with side gates deposited in the same step. For the device presented here, the end of the upper left gate broke off during processing. However, the device could be tuned well without it.

Master equations. The master equations (used for Fig. 1b) consider states with fixed total parity, composed of the combined parity of quasiparticles in the thermalized continuum and the 0,1 , or 2 quasiparticles in the bound state (see Supplementary Information).

Free energy model. Even and odd partition functions in equation (2), $F_{\mathrm{o}}-F_{\mathrm{e}}=-k_{\mathrm{B}} T \ln \left(Z_{\mathrm{o}} / Z_{\mathrm{e}}\right)$, can be written as sums of Boltzmann factors over respectively odd and even occupancies of the isolated island. For even occupancy,

$$
Z_{\mathrm{e}}=1+\sum_{i \neq j} \mathrm{e}^{-E_{i} / k_{\mathrm{B}} T} \mathrm{e}^{-E_{j} / k_{\mathrm{B}} T}+\cdots
$$

where the first term stands for zero quasiparticles, the second for two (at energies $E_{i}$ and $E_{j}$ ), and additional terms for four, six, and so on. $Z_{\mathrm{o}}$ similarly runs over odd occupied states. Rewriting these sums as integrals over positive energies yields

$$
F_{\mathrm{o}}-F_{\mathrm{e}}=-k_{\mathrm{B}} T \ln \tanh \int_{0}^{\infty} \mathrm{d} E D(E) \ln \operatorname{coth}\left(E / 2 k_{\mathrm{B}} T\right)
$$

where $D(E)$ is the density of states of the HQD,

$$
D(E)=\rho_{\mathrm{BCS}}(E)+\frac{1}{2} \rho_{0}^{+}(E)+\frac{1}{2} \rho_{0}^{-}(E)
$$

We take $\rho_{\mathrm{BCS}}(E)$ to be a standard BCS density of states,

$$
\rho_{\mathrm{BCS}}(E)=\frac{\rho_{\mathrm{Al}} V E}{\sqrt{E^{2}-\Delta(B)^{2}}} \theta(E-\Delta)
$$

( $\theta$ is the step function), and $\rho_{0}$ to be a pair of Lorentzian-broadened spinful levels symmetric about zero,

$$
\rho_{0}^{ \pm}(E)=\frac{\gamma / 2 \pi}{\left(E-E_{0}^{ \pm}\right)^{2}+(\gamma / 2)^{2}}+\frac{\gamma / 2 \pi}{\left(E+E_{0}^{ \pm}\right)^{2}+(\gamma / 2)^{2}}
$$

Zeeman splitting of the bound state and pair-breaking by the external magnetic field are modelled with the equations

$$
\begin{aligned}
& E_{0}^{ \pm}(B)=\frac{\Delta(B)}{\Delta} E_{0} \pm \frac{1}{2} g \mu_{\mathrm{B}} B \\
& \Delta(B)=\Delta \sqrt{1-\left(\frac{B}{B_{\mathrm{c}}}\right)^{2}}
\end{aligned}
$$

where $E_{0}$ is the zero-field state energy and $\Delta$ is the zero-field superconducting gap. In the event that a bound state goes above the continuum, $E_{\mathrm{s}}^{+}>\Delta(B)$, we no longer include the state in the free energy. Equation (3) was integrated numerically to obtain the theoretical curves in Fig. 4.

Equations (4) and (5) are reasonable, provided the lower spin-split state remains at positive energy, $E_{0}^{-}>0$. For sufficiently large $B_{c}$, the bound state will reach zero energy, resulting in topological superconductivity and Majorana modes, the subject of future work. 